\title{
Clinical Transformation: The Key to Green Nephrology
}

\author{
Andrew Connor ${ }^{\mathrm{a}}$ Frances Mortimer $^{\mathrm{b}}$ Charles Tomson ${ }^{\mathrm{c}}$

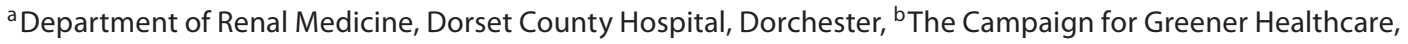 \\ Oxford, and 'Southmead Hospital, Bristol, UK
}

\section{Key Words}

Disease prevention - Green nephrology •

Lean service delivery - Low-carbon technologies •

Patient empowerment

\begin{abstract}
Climate change represents a major global public health threat. The very provision of healthcare itself has a significant untoward effect on the environment, to which kidney care is likely to contribute disproportionately. In this article we describe the four principles we believe will underpin a successful transformation to lower carbon kidney care: disease prevention, patient empowerment, lean service delivery and the preferential use of low-carbon technologies. We illustrate their application and their co-benefits, such as improvements in patient care and reductions in cost, with examples.

Copyright $\odot 2010$ S. Karger AG, Basel
\end{abstract}

\section{Introduction}

The evidence that climate change is happening, and that human activity is driving it, is now incontrovertible [1]. A recent Lancet/University College London Commission considered climate change to be 'the biggest global health threat of the 21st century' [2]. However, the very provision of healthcare itself also has a significant unto- ward environmental impact. The carbon footprint of the NHS England, for example, is 18.6 million tonnes of carbon dioxide per year in England alone [3], and kidney care is likely to contribute disproportionately to this.

There are a number of reasons why the healthcare sector must act to reduce its emissions [4], and many configurations to how this might be achieved. Carbon reduction strategies often focus on energy use within the built environment. However, this contributes only $22 \%$ of NHS emissions [3] - an indication that, whilst such initiatives are both visible and important, they represent only a small part of the solution. Instead, the current systems and practices used to provide healthcare must be transformed. We describe four principles to underpin a clinical transformation to lower carbon kidney care, illustrating their application and co-benefits (e.g. improvements in patient care and reductions in cost) with examples.

\section{The Principles of a Transformation to Sustainable Clinical Practice}

\section{Disease Prevention}

The financial and carbon costs associated with healthcare are increasing in line with improvements in our ability to diagnose and treat disease. For example, the recent implementation of widespread routine reporting of eGFR across most healthcare services was followed by a rise in the number of referrals to nephrology services, and an

\section{KARGER}

Fax +4161306 1234

E-Mail karger@karger.ch

www.karger.com (c) 2010 S. Karger AG, Basel

$1660-2110 / 10 / 1163-0200 \$ 26.00 / 0$

Accessible online at:

www.karger.com/nec
Dr. Andrew Connor

Department of Renal Medicine, Dorset County Hospital

Williams Avenue, Dorchester DT1 1JY (UK)

E-Mail andrew.connor@kintoa.org 
increase in their workloads (albeit balanced by a reduction in late referrals). We must now look further upstream, to the prevention of disease, to realise further health benefits and redress the carbon and financial costs of the care we provide. Both in individual patient care and through broader advocacy, kidney care should aim to tackle underlying causes of disease - modifying the social, economic and environmental determinants of health. The identification of effective strategies to achieve this must become a research priority.

\section{Patient Empowerment and Self-Care}

Patient empowerment is an established component of the successful management of chronic disease, but its benefit in facilitating lower-carbon care is now also being recognised. Empowered patients are both proactive and reactive in the management of their conditions, preventing and responding to the challenges they present. Such patients, for example those undertaking home-based renal replacement therapies, require less input from healthcare professionals, reducing the financial and carbon costs of the care they receive. Strategies to empower patients, and to teach them when and how to access healthcare, are vital to the transformation to low-carbon healthcare. Innovative services which appear to empower patients, such as telephone clinics, should be combined with more established patient empowerment tools such as RenalPatientView. Further research to validate the benefits to the environment and patients of such services should be supported.

\section{Lean Service Delivery}

Wherever possible, care should be simplified. Reductions in the number of lower-value activities, and their associated environmental impacts, can be achieved through the sympathetic design of care pathways and also through considered clinical decision-making in the selection of interventions and follow-up. Higher value activities should be championed, and supported by strategies to reduce travel emissions (such as virtual consultations and electronic records).

Lean thinking has formed the backbone of diverse initiatives to meet many challenges within the healthcare setting. Examples include the amelioration of the risk to patient safety through improved standardisation, and reductions in waiting times in emergency departments and outpatient services. Lean methodology, and the reduction of waste in particular, is also attractive to those wishing to reduce the emissions associated with healthcare systems (in which duplication is common) as it also offers lower financial costs, improved patient experience and a lower risk of harm.

\section{Preferential Use of Low-Carbon Technologies}

The inclusion of sustainability measures in the evaluation of medical technologies will allow clinicians and patients to choose clinically effective treatments with the optimal environmental profiles. Clinicians should not be expected to make such decisions on an individual patient basis. Instead, these considerations must be incorporated into the policies determining the provision of kidney care, such as the National Service Framework for renal services in the UK, and should be supported by a broadening of the spectrum of future research.

\section{Carbon Reduction as Part of Environmental Sustainability}

Although climate change presents the most urgent and devastating environmental threat to global health, it is just one consequence of human activity on the Earth's physical and biological systems, and serves as an alarming reminder that the environmental impact of our activities (including healthcare) are of direct relevance to human health and wellbeing. Therefore, in tandem with reducing carbon emissions, all aspects of the environmental impact of kidney care should also be addressed. These include the consumption of water and of non-renewable resources, environmental pollution, and impacts on biodiversity.

\section{Opportunities to Improve the Sustainability of Dialysis}

More than 1 million patients receive haemodialysis worldwide. In the UK, $41.1 \%$ of all patients receiving renal replacement therapy are treated by centre-based haemodialysis [5], necessitating lifelong, three times weekly return journeys (usually by car). Haemodialysis also uses large amounts of energy and mains water, and produces large volumes of plastic and packaging waste. Here we describe just a few of the many opportunities that exist to reduce the environmental impact of haemodialysis.

\section{Water Conservation}

More than one third of the world's population now live in water-stressed countries. The provision of haemodialysis uses water, a finite natural resource, in vast quanti- 
ties. A patient receiving dialysis for $4 \mathrm{~h}$, with a target dialysate flow rate of $500 \mathrm{ml} / \mathrm{min}$, will require 120 litres of dialysate. In most facilities, this is drawn from the mains water supply and the purification process commonly employs reverse osmosis to remove residual salts. Reverse osmosis systems reject around two thirds of the water presented to them. Accounting for the water necessary to prime and cleanse the dialysis machine, a total volume approaching 500 litres of mains water is required per patient per 4-hour session.

The 'reject water' from the reverse osmosis system is high-grade grey water that usually meets the biochemical criteria for potable water (although it remains legally unacceptable for drinking) [6]. It poses no infection risk as it does not come into contact with either the patient or the dialyser. However, in most haemodialysis services worldwide, and in all but one in the UK, large volumes of reject water are simply lost to drain. The simple strategies, implemented by a minority of individual units worldwide, to recycle reject water for alternative uses (such as laundry, sanitation and low-pressure boiler feed) have been proven to be cost-effective [6], and must now be widely adopted.

\section{Home versus In-Centre Haemodialysis}

Preliminary work suggests that home haemodialysis is less damaging to the environment than in-centre haemodialysis [7]. Home haemodialysis is also more cost-effective and offers an improved quality of life. Whilst robust evidence of improved patient outcomes by way of randomised controlled trials is lacking (primarily as a result of the difficulties presented by the inherent selection bias), many clinicians are increasingly convinced of the superiority of home haemodialysis and very few would argue it to be genuinely inferior. However, the uptake of home haemodialysis varies dramatically between and within countries, and has declined considerably in many regions [8]. Significant expansion of home haemodialysis is likely to be possible in many countries, in part through embracing evolving technologies. A better understanding of the environmental impact of in-centre and home therapies is therefore important.

There has been only one study to date [7]. Methodological difficulties in analysing the absolute carbon footprints of these two modalities prompted the adoption of a comparative approach in which quantification of the differences in their environmental impacts was attempted. Data relating to transport, energy and water use was acquired and home haemodialysis was found to produce less emissions ( 0.207 tonnes of carbon dioxide) than in- centre haemodialysis (1.404 tonnes) per patient per year. This discrepancy was predominantly explained by the absence of emissions related to patient travel in home haemodialysis. However, these findings should be interpreted with caution given that the study was underpowered to ascertain differences in water consumption and that several potentially significant sources of emissions were omitted altogether. Although these omissions included heating and lighting, perhaps the most important was that of the energy embedded into the haemodialysis machines and other medical equipment during the manufacturing process. Whilst in-centre machines might be used up to three times per day, the machines of home haemodialysis patients may only be used three times weekly. This discrepancy, in conjunction with their high but commensurate embedded energies, is likely to have implications for the overall emissions attributable to the two modalities. Further studies are required to confirm whether the reduction in patient travel allowed by home haemodialysis truly translates to a lower environmental impact than in-centre haemodialysis, and should also account for home haemodialysis patients potentially opting to dialyse more frequently (using more consumables) and for longer (using more water and electricity). Such work may strengthen the movement towards increasing the uptake of this modality, with the potential benefits to patients and the environment increasing as the numbers of patients receiving haemodialysis continue to rise.

\section{Further Opportunities to Reduce}

Haemodialysis-Associated Emissions

Heat exchangers, which recycle heat from the dialysis effluent into the warming of the incoming dialysate, offer considerable energy savings but are not yet fitted as standard to all haemodialysis machines. The use of central delivery systems to provide concentrated dialysate acid reduces both dialysate wastage and the plastic waste derived from dialysate containers (which may prove difficult to recycle as the possibility of residual acid often discourages local recycling facilities). Further environmental savings are possible if this concentrate is delivered in a 1:44 concentration (the maximum that most machines can proportion), thereby reducing the emissions related to the transportation of large volumes of liquid. This benefit would be greater still if the dialysate components were to be delivered in powder form and reconstituted on site, however availability and concerns around composition errors and contamination presently discourages many units from this option. Clinicians should also be aware of the considerable increases in reject water production 
(around 72 litres per treatment) that arise from increasing the dialysate flow rate from 500 to $800 \mathrm{ml} / \mathrm{min}$, and the lack of firm evidence that this strategy actually increases delivered dialysis dose at all.

\section{Dialyser Reuse: An Area of Uncertainty}

Dialyser reuse has been primarily driven by financial considerations and is most commonplace in the USA. A further advantage, the prevention of first-use syndromes through the improvement in blood-membrane biocompatibility, has become less significant as more biocompatible membranes have become available. The health concerns around infection risk and exposure to residual germicide from the decontamination process are sufficiently small that dialyser reuse is estimated to be undertaken in approximately $40 \%$ of dialysis facilities in the USA, although rates are falling.

Dialyser reuse produces less waste than the practice of single-use, which has been estimated to contribute over 4,000 tonnes of polymer waste annually in the USA [9]. Such clinical waste must undergo incineration, a form of waste management that is preferable only to landfill in terms of its environmental impact. Dialyser reuse also reduces the amount of dialyser-related packaging waste. Yet the sustainability of dialyser reuse is unclear. The use of germicides, such as formaldehyde and peracetic acid, in the decontamination process raises environmental concerns as it produces increased liquid waste (heated water, chemicals and disinfectants) and chemical vapours (such as hypochloric acid). The disinfection process also increases the use of disposable waste (such as masks, gloves, aprons and test strips). As both single-use practice and more frequent dialysis regimes are increasingly common worldwide, there is a need for further advances in dialysis waste management.

\section{Clinical Waste Management}

Much of the waste generated from dialysis products and their packaging falls into the category of clinical waste. A single haemodialysis session produces approximately $2.5 \mathrm{~kg}$ of solid clinical waste, of which $38 \%$ is plastic [10]. This amounts to an estimated $390 \mathrm{~kg}$ per year, which compares with $617 \mathrm{~kg}$ per year produced by a patient undertaking a standard continuous ambulatory peritoneal dialysis regime. Neither estimate accounts for the substantial cardboard and polythene waste that is often associated with packaging. Clinical waste is subject to the Environmental Protection Act of 1990 in the UK and must be incinerated. This expensive process produces further emissions, including dioxins, carcinogenic compounds produced on incineration of PVC. Renal services must develop sustainable waste management strategies. Effective segregation at source is vital and requires staff education and extra space, to ensure that non-clinical waste (e.g. packaging) is not treated as clinical waste but, where possible, recycled.

\section{Opportunities within the Outpatient Setting}

Many clinicians, faced with the challenge of providing increasingly high standards of care to rising numbers of patients with greater expectations, are exploring virtual care models. These offer patients and clinicians a number of benefits including enhanced access to healthcare, heightened continuity of care, patient empowerment and time savings. There are also clear benefits to the patients and the environment from the reduction in patient travel. Virtual care is not appropriate when formal physical examination is a likely necessity, but is well suited to the provision of follow-up for chronic disease, which forms much of kidney care. Yet whilst virtual care has become an established method of reducing the burden on primary care and emergency departments, relatively little information exists on its use within kidney care. Videoconferencing can facilitate routine consultations between nephrologists and dialysing patients [11]. Non-comparative studies have demonstrated the value of telemedicine in both haemodialysis and peritoneal dialysis, suggesting benefits in terms of improved access, cost and travel savings for patients [12]. Informal patient feedback from a UK centre providing routine follow-up to transplant recipients through telephone consultations also highlights the way such models can engender empowerment amongst patients. Provision must be made within local funding models, particularly those which incentivise activity, to support virtual care in its different guises. Further research should focus on the safety and benefits of such systems.

\section{The Interface with the Renal Industry}

The interface between the renal industry and those delivering healthcare represents an important opportunity for improving sustainability within kidney care. Procurement is a case in point. Renal units should be encouraged to incorporated sustainability criteria into all pro- 
curement contracts. These must be afforded appropriate weighting, and must ensure that a genuine commitment to sustainability exists throughout the supply chain. They should be measurable and relate to both the organisation and product. Such efforts will incentivise improvements amongst competitors. Dialogue between the industry and healthcare providers is also vital at each stage of the product life cycle; for example, are all the components of a product routinely utilised? Will suppliers remove packaging for recycling at delivery?

\section{Reducing the Environmental Impact of Medicines}

Procurement contributes $60 \%$ of the total NHS emissions, with pharmaceuticals and medical equipment being primarily responsible [3]. This figure unmasks the indirect emissions related to the manufacturing process. Sustainable procurement, on a global scale, will help to drive down these emissions but the process will be slow. Influencing the consumption of medications may appear more immediately feasible. Up to $40 \%$ of medications prescribed to those with chronic conditions are never taken [13]. This figure may be even higher amongst the renal population, in whom polypharmacy and complicated regimes are commonplace. These medications are rarely returned to the pharmacy and, in any case, the Royal Pharmaceutical Society of Great Britain dictates that all returned medications must be destroyed (as the quality can no longer be guaranteed), entailing enormous financial and environmental costs.

Attention must therefore focus on reducing drug wastage, in which clinicians can play a major role through three simple measures. Firstly, they should identify and modify poor compliance. Secondly, prescribers should strive to reduce inequivalence, whereby different items on the same prescription are allotted differing durations. This requires patients to manage their repeat prescriptions. Some patients will struggle to order only the items they require and instead will choose to re-order all the items regardless of their existing stock, leading to drug hoarding. Finally, the prescription of excessive quantities should be avoided, as this leads to greater wastage when changes are made. This is particularly important when medications are first commenced and changes are most likely. Reducing the need for medications through the adoption of healthier, lower-carbon lifestyles will also help to reduce the medication-associated emissions.

\section{The Impact of Environmental Pollution on Kidney Care}

Whilst we have focused on the environmental gains possible from changes to the way kidney care is provided, the converse - that kidney care will benefit from reductions in environmental pollution made outside of the healthcare setting - is also true. Solvents, such as hydrofluorocarbons, are man-made chemicals with high global warming potentials that are used to manufacture products such as paints, adhesives, automotive electronics, computers and medical equipment. Occupational solvent exposure hastens the progression of existing CKD and may also contribute to its initiation [14]. Similarly, heavy metals such as cadmium, mercury and lead, are non-biodegradable, have long half-lives, and represent a major environmental hazard. They are also nephrotoxic at very low doses, through both acute and chronic exposures, and cause varying degrees of nephropathy from tubular dysfunction to end-stage renal disease. These examples illustrate how public health strategies to limit environmental pollution will reduce the burden of kidney disease.

Even inside kidney care, direct exposure to man-made chemicals in clinical products may be harming patients. Dialysis products made from polyvinylchloride (PVC) typically contain the softener di-(2-ethylhexyl)phthalate (DEHP), which is toxic to reproduction according to the EU Directive 67/548/EEC on Classification and Labelling of Dangerous Substances. The 2008 EU Risk Assessment on DEHP [15] highlighted the need to limit risks to consumers from medical equipment used in long-term haemodialysis. PVC/DEHP-free plastic dialysis products are now available and the wholesale elimination of DEHP could be achieved through alterations to renal procurement policies.

\section{The Health Co-Benefits of Low-Carbon Lifestyles}

The adoption of low-carbon lifestyle initiatives should be encouraged both at the level of individual patient care and through broader advocacy. Such strategies might include the promotion of active travel (walking, cycling) and dietary modifications (reduced consumption of red meat and increased consumption of locally sourced foods). The health co-benefits of these strategies will contribute to reductions in hypertension, obesity and diabetes - predominant drivers of the epidemic of chronic kidney disease. 


\section{The Cost of Greening Kidney Care}

The cost of limiting emissions to below 550 parts per million carbon dioxide equivalents has been estimated at $1 \%$ of the global per capita consumption per year, whilst allowing unchecked emissions may cost the world up to $20 \%$ of global consumption per year, albeit not realised for up to 200 years [16]. Thus the argument that measures to reduce emissions will cripple the world's economy, and that further economic growth should precede their introduction, is weak and such measures should be taken now. But, given that the current economic climate will ensure that healthcare systems face unprecedented reductions in resources (or, at the very least, diminished expansion in the face of ongoing increases in demand), should they too really invest in green technologies now?

We suggest that they should. It should be remembered that the aforementioned economic arguments apply less well to most healthcare systems, in which increased activity is dependent upon economic growth rather than a driver for it. Indeed, the spiralling costs of the healthcare system in the USA are considered to be a major threat to the nation's economy. Instead, with lean principles and the removal of waste at the heart of many green initiatives within kidney care, the potential for a profitable return on an investment is real. For example, the financial investment required to incorporate reject water conservation methodology into the design of a satellite dialysis facility in the UK was returned during its first few months of operation, whilst the potential annual saving from its continued use is estimated to be four-fold greater than the one-off investment cost. We argue, therefore, that considered investment in green initiatives is not only affordable, but represents sound business (and environmental) sense.

\section{Conclusion}

Healthcare organisations, and renal services in particular, must act to reduce the environmental impact of their own practice. Many of the necessary interventions are supported by strong business cases; reducing financial costs (especially as fuel prices are predicted to continue to rise), complying with legislation, improving staff morale, and advertising social responsibility credentials. Shared learning and determination of best practice is both essential and underway (http://www.greenerhealthcare.org/nephrology-resources).

\section{References}

1 Solomon S, Qin D, Manning M, et al (eds): Climate Change 2007: The Physical Science Basis, Contribution of Working Group I to the Fourth Assessment Report of the Intergovernmental Panel on Climate Change. Cambridge, Cambridge University Press, 2007.

-2 Costello A, Abbas M, Allen A, et al: Managing the health effects of climate change. Lancet 2009;373:1693-1733.

3 Sustainable Development Commission (2008): NHS England Carbon Emissions: Carbon Footprinting Study. London, Sustainable Development Commission, September 2008.

4 Connor A, Tomson C, Mortimer F: Renal medicine can take the lead in greener healthcare. Br J Ren Med 2009;14:19-22.

5 The Renal Association, UK Renal Registry, Eleventh Annual Report, Bristol, UK, UK Renal Registry, December 2008. Available at: http://www.renalreg.com/Reports/2008. html (accessed December 12, 2009).
-6 Agar JWM, Simmonds RE, Knight R, Somerville CA: Using water wisely: new affordable, and essential water conservation practices for facility and home haemodialysis. Hemodial Int 2009; 13:32-37.

7 James R: Dialysis and the environment: comparing home- and unit-based haemodialysis. J Ren Care 2007;33:119-123.

-8 MacGregor MS, Agar JWM, Blagg CR Home haemodialysis - international trends and variation. Nephrol Dial Transplant 2006;21:1934-1945.

$\checkmark 9$ Upadhyay A, Sosa MA, Jaber BL: Single use versus reusable dialyzers: the known unknowns. Clin J Am Soc Nephrol 2007;2: 1079-1086.

10 Hoenich NA, Levin R, Pearce C: Clinical waste generation from renal units: implications and solutions. Semin Dial 2005;18: 396-400.

11 Whitten P, Buis L: Use of telemedicine for haemodialysis: perceptions of patients and healthcare providers. J Telemed Telecare 2008;14:75-78.
12 Jennett PA, Afflect Hall L, Hailey D, et al: The socio-economic impact of telehealth: a systematic review. J Telemed Telecare 2003; 9:311-320.

13 Sabaté E (ed): Adherence to Long-Term Therapies: Evidence for Action. Geneva, WHO, 2003

$\checkmark 14$ Jacob S, Héry M, Protois JC, Rossert J, Stengel B: Effect of organic solvent exposure on chronic kidney disease progression: the GNProgress cohort study. J Am Soc Nephrol 2007;18:274-281.

15 European Union Risk Assessment Report Bis-(2-ethylhexyl)phthalate (DEHP), CAS No. 117-81-7 EINECS No. 204-211-0. Office for Official Publications of the European Communities, 2008. http://ecb.jrc.ec.europa.eu/documents/Existing-Chemicals/ RISK_ASSESSMENT/REPORT/dehpreport042.pdf.

16 Stern N: The Economics of Climate Change: The Stern Review. Cambridge, Cambridge University Press, 2007. 


\section{Editorial Comment}

M. El Nahas, Sheffield

Andrew Connor and his colleagues bring ecology to nephrology in a challenging review on 'green nephrology'. Clearly, nephrology practice cannot avoid the tide of green practices and energy conservation. Energy-efficient dialysis facilities may prove to be essential; strategies based on combined changes practice in haemodialysis including modifying hard- and software to minimise energy expenditure, fuel switching, transport and behavioural changes can potentially save considerable carbon dioxide $\left(\mathrm{CO}_{2}\right)$. In fact, energy conservation is also likely to impact on general and population health including causes and complications of CKD; household energy interventions have potential for important cobenefits in pursuit of health and climate goals [1]. Nephrologists cannot avoid the challenge of sustainability.
This is likely to be all the more timely in view of growing service demands based on an expanding CKD and ESRD population in the face of restricted and diminishing funding. The challenge is similar to other major public health issues in that it needs to be clearly defined, evidence gathered, theories developed, alliances built, policies formulated and actions taken [2]. Facts need to inform trends!

\section{References}

Wilkinson P, Smith KR, Davies M, Adair H, Armstrong BG, Barrett M, Bruce N, Haines A, Hamilton I, Oreszczyn T, Ridley I, Tonne C, Chalabi Z: Public health benefits of strategies to reduce greenhouse-gas emissions: household energy. Lancet 2009;374:1917-1929.

McCartney G, Hanlon P: What can health professionals contribute to the challenge of sustainability? Public Health 2009;123:761-764. 